\title{
Effect of one gold salt, of betamethasone, and of aspirin on the chemotaxis of human neutrophils measured in vitro
}

\author{
ALAIN PÉCOUD, ANNETTE LEIMGRUBER, AND PHILIPPE C. FREI \\ From the Division of Immunology and Allergy, Department of Medicine, CHUV, CH-1011 Lausanne, \\ Switzerland
}

SUMMARY The effects of 1 gold salt, of betamethasone, and of acetylsalicylate on the chemotaxis of human neutrophils were compared in normal volunteers by means of a modified Boyden chamber. The gold salt inhibited chemotaxis by reducing both cell migration capacity and the capacity of plasma to attract the cell. Betamethasone reduced cell migration capacity, but not the chemoattractant capacity of plasma. Acetylsalicylate had no effect on these functions.

Various drugs are known to be effective in reducing inflammatory lesions in patients suffering from rheumatoid arthritis. Among these, aspirin, gold salts and corticosteroids are commonly used at some time in the course of the disease. However, their anti-inflammatory effect is still poorly understood. Apart from their effect on prostaglandin metabolism they could interfere with lymphocyte response and the major functions of granulocytes and macrophages, i.e., chemotaxis, phagocytosis, and killing capacity.

The chemotaxis of neutrophils is defined as an active and directed migration of the cells towards a gradient of substances which attract the cell to the site of inflammation (Keller et al., 1977). It occurs in an early phase of the cascade of inflammatory events which finally produce the clinical signs of arthritis. Inhibition of chemotactic function may therefore result in an important reduction of the inflammatory lesions.

It is not clearly established what effects the antiinflammatory drugs under study may have on the chemotactic function of neutrophils. The effects of steroids (Ward, 1971; Peters et al., 1972; Sneiderman and Wilson, 1975; Majeski and Alexander, 1976; Shea and Morse, 1978) and those of aspirin (Ward 1966; Magliulo et al., 1977) are still a subject of controversy. Baum (1975) has reported that gold salts do not affect chemotaxis of neutrophils, but recently a definite impairment of this function was

Accepted for publication 2 March 1979

Correspondence to P. C. Frei, Department of Medicine, CHUV, CH 1011 Lausanne, Switzerland. observed by Mowat (1978) both in normal controls and in patients with rheumatoid arthritis.

The purpose of this study was to compare the effects of these 3 commonly used anti-inflammatory drugs on chemotactic function measured in vitro. In particular we investigated their effect on both components of this function: the ability of neutrophils to migrate and the ability of plasma to attract the cell.

\section{Materials and methods}

\section{LE UCOCYTES}

$10 \mathrm{ml}$ of venous blood was drawn from healthy blood donors and heparinised. It was added to $5 \mathrm{ml}$ of $6 \%$ dextran 250 (Pharmacia) and allowed to settle at room temperature for 1 hour. The supernatant was then centrifuged and the leucocytes were resuspended in a phosphate buffer, $\mathrm{pH} 7 \cdot 2$, containing $2 \%$ human serum albumin.

\section{CHEMOTAXIS ASSAY}

Leucocyte chemotaxis was studied in a modified Boyden chamber (Boyden, 1962), which was described in detail elsewhere (Frei et al., 1974). It consisted of 2 compartments separated by a filter (Millipore; pore diameter $3 \mu \mathrm{m}$ ). The upper compartment was filled with buffer containing $10^{6}$ leucocytes. The lower compartment was filled with $1.5 \mathrm{ml}$ of diluted autologous plasma. The plasma was made chemoattractive by preincubation for $\mathbf{3 0}$ minutes at $37^{\circ} \mathrm{C}$ with $50 \mu \mathrm{l}$ of heat aggregated gammaglobulin. This compound was obtained by previously 
heating commercial gammaglobulin (Globuman Berna $160 \mathrm{mg} / \mathrm{ml}$ ) for 60 minutes at $64^{\circ} \mathrm{C}$.

In some experiments (migration without chemoattractant, or 'random migration') buffer instead of activated plasma was introduced into the lower compartment.

Cells were allowed to migrate for $2 \frac{1}{2}$ hours at $37^{\circ} \mathrm{C}$. The 2 compartments were then separated. The filters were fixed and stained, and the total number of neutrophils remaining on the lower surface of the filter was counted. The lower compartment was centrifuged, and its bottom was examined-from below with an inverted microscope equipped with a graduated ocular. The total number of leucocytes which had fallen on to the bottom of the lower compartment was counted. Chemotaxis was expressed as the total number of migrated cells, obtained by adding the number of cells still adhering to the lower surface of the filter to the number of cells which had fallen on to the bottom of the chamber.

Each individual experiment was performed in 3 parallel chambers. The coefficient of variation (SD of the differences between 3 triplicates $\times 100$ divided by the mean) calculated on 25 randomly selected experiments was $4 \cdot 5 \%$.

When activated plasma had been used as the chemoattractant, $167470 \pm 11600$ (mean \pm SEM; $\mathrm{n}=139$; range $99760-219220$ ) cells had migrated in the control experiments (without drug). So approximately $10-20 \%$ of the cells introduced into the upper compartment were found in the lower compartment after migration. When activated plasma was replaced by buffer alone (migration without chemoattractant) $1530 \pm 106$ (mean \pm SEM; $n=$ 134; range 740-2320) cells had migrated in the control experiment (without drug), i.e., approximately per 1000 of the cells introduced into the upper compartment.

\section{DR UGS}

The effect of the drugs was measured in three different ways.

Drug added to cells. The cell suspension was separated into 4 aliquots which were then preincubated for 30 minutes at $37^{\circ} \mathrm{C}$ respectively in buffer alone and in buffer containing 3 concentrations of the drug under examination. Immediately after preincubation the cell suspension was introduced into the upper compartment and the cells were allowed to migrate. $\left(A_{1}\right)$ In some of the other experiments in this series the cells were washed once $\left(A_{2}\right)$, that is, the cell suspension was centrifuged after incubation, the supernatant was discarded, and the cells resuspended in drug-free buffer. They were then adjusted to the usual yield of $10^{6}$ cells and finally introduced into the upper compartment and allowed to migrate. In experiment $A$ no drug was added to the lower compartment.

Drug added to plasma. The plasma was separated into 4 aliquots, which were diluted 1:5 with buffer alone or with buffer containing 3 concentrations of the drug under examination. Incubation with drug and aggregated gammaglobulin was carried out simultaneously. In experiments with buffer instead of plasma no aggregated gammaglobulin was used. After incubation for 30 minutes at $37^{\circ} \mathrm{C}$ activated plasma or buffer was introduced into the lower compartment. No drug was added to the leucocyte suspension in the upper compartment.

Drug added to cells and plasma. Cells and autologous plasma were preincubated separately but simultaneously in the same conditions as for experiments $A$ and $B$ and then introduced into the upper and lower compartments respectively.

Cell viability was tested by the method of trypan blue dye exclusion. In all experiments the highest percentage of dead cells found was $4 \%$.

The following drugs were used: (1) a saline solution $(2 \cdot 5 \mathrm{mg} / \mathrm{ml})$ of aurothiopropanol sodium sulphonate containing $30 \%$ gold (Allochrysine, Lumière, France); (2) a saline solution $(4 \mathrm{mg} / \mathrm{ml})$ of betamethasone disodium phosphate (Celestone, Schering, USA); (3) an aqueous solution $(250 \mathrm{mg} / \mathrm{c}$ $\mathrm{ml}$ ) of lysine acetylsalicylate containing $50 \%$. acetylsalicylic acid (Aspegic, Egic, France). Drugs were further diluted in buffer in order to obtain final concentrations equivalent to the usual therapeutic levels.

\section{ANALYSIS OF THE RESULTS}

Because of large differences between donors in the control experiments (migration without a drug) we expressed the results as a percentage of the control value, that is, the absolute number of migrating cells after preincubation with drug $\times 100$ divided by the absolute number of migrating cells in the same subject after preincubation with buffer. Means of triplicates were used for statistical analysis by Student's $t$ test for paired or unpaired data when appropriate. The correlation coefficient of the log dose/response curve was calculated by means of the least square method, the difference between the absolute number of cells preincubated with drug and those preincubated with buffer being plotted against $\log _{10}$ of the drug concentration.

\section{Results}

CHEMOTAXIS (MIGRATION TOWARD CHEMOATTRACTANT)

Gold salt (Table 1). Preincubation of the leucocytes with aurothiopropanol (experiment A) decreased the 
Table 1 Effect of sodium aurothiopropanol sulphonate on the chemotaxis of neutrophils

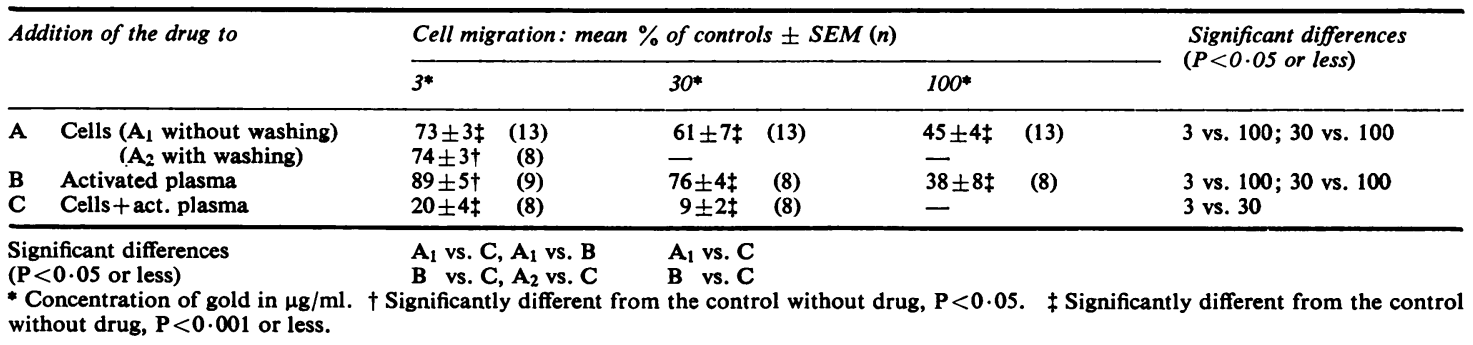

chemotactic response of the cells. This reduction in chemotactic reactivity was dose-dependent as shown on a log dose/response curve (Fig. 1) $(r=0 \cdot 479$, $\mathrm{n}=39, \mathrm{P}<0 \cdot 01)$. Washing the cells after incubation did not suppress the reduction induced by preincubation with the drug. Since the washed neutrophils were resuspended in drug free medium, no drug could diffuse through the filter and induce an effect on the plasma. This seems to indicate that the drug acted on the cell itself. A similar inhibition was induced by preincubating the plasma with the same drug concentrations (experiment B). Preincubating

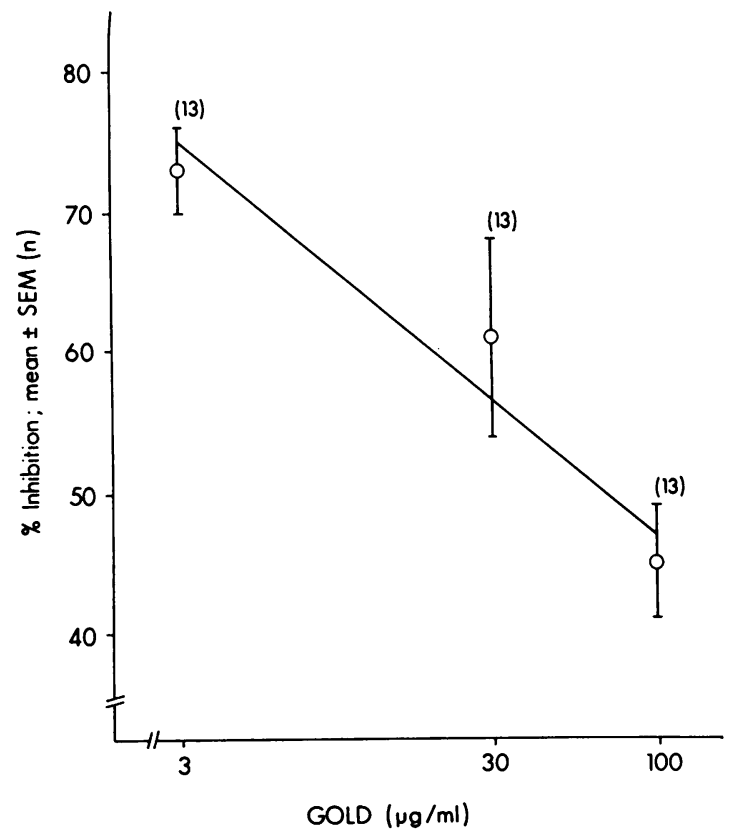

Fig. 1 Chemotaxis of neutrophils after incubation with a gold salt (Na aurothiopropanol sulphonate); the inhibition of migration (vertical scale) is expressed as $\%$ of the cell migration after incubation in a drug-free medium. Numbers in parentheses represent the number of experiments both cells and plasma with 3 and $30 \mu \mathrm{g} / \mathrm{ml}$ gold (experiment C) decreased the migration to $20 \%$ and $9 \%$, which is definitely more than observed in experiments $A$ and $B$, where only 1 of the 2 compartments was incubated with the drug.

Betamethasone (Table 2). Neutrophil chemotaxis was decreased to 85 and $66 \%$ when the neutrophils were incubated with the 2 highest concentrations of betamethasone i.e., 0.01 and $0.1 \mu \mathrm{g} / \mathrm{ml}$ (experiment A). Again, this effect was dose-dependent (Fig. 2) $(r=0.653, n=33, P<0.001)$ and could not be eliminated by washing. Incubating the plasma

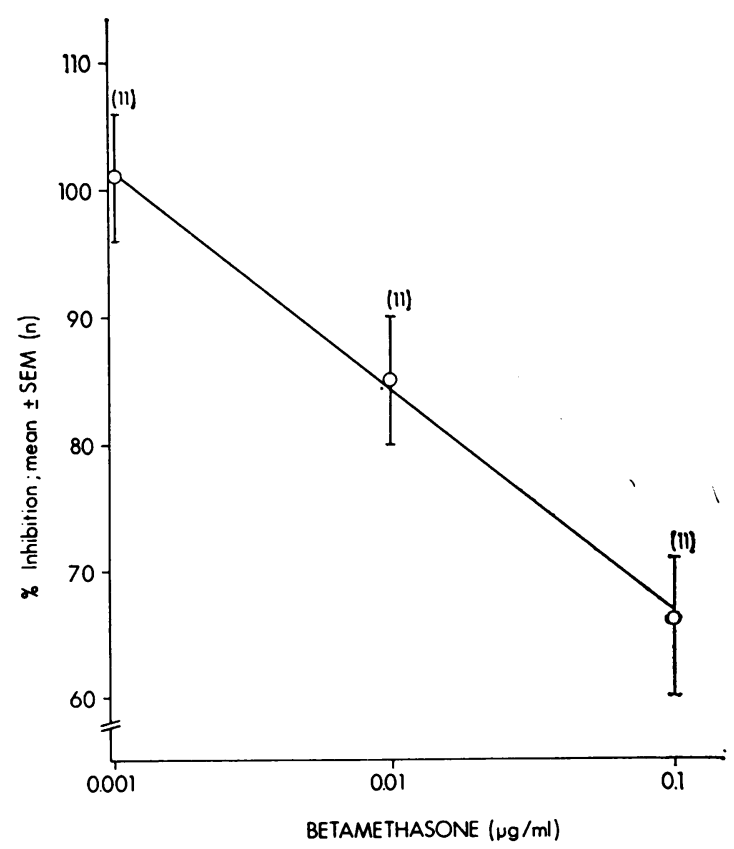

Fig. 2 Chemotaxis of neutrophils after incubation with betamethasone; the inhibition of migration (vertical scale) is expressed as $\%$ of the cell migration after incubation in a drug free medium. Numbers in parentheses represent the number of experiments 
Table 2 Effect of betamethasone on the chemotaxis of neutrophils

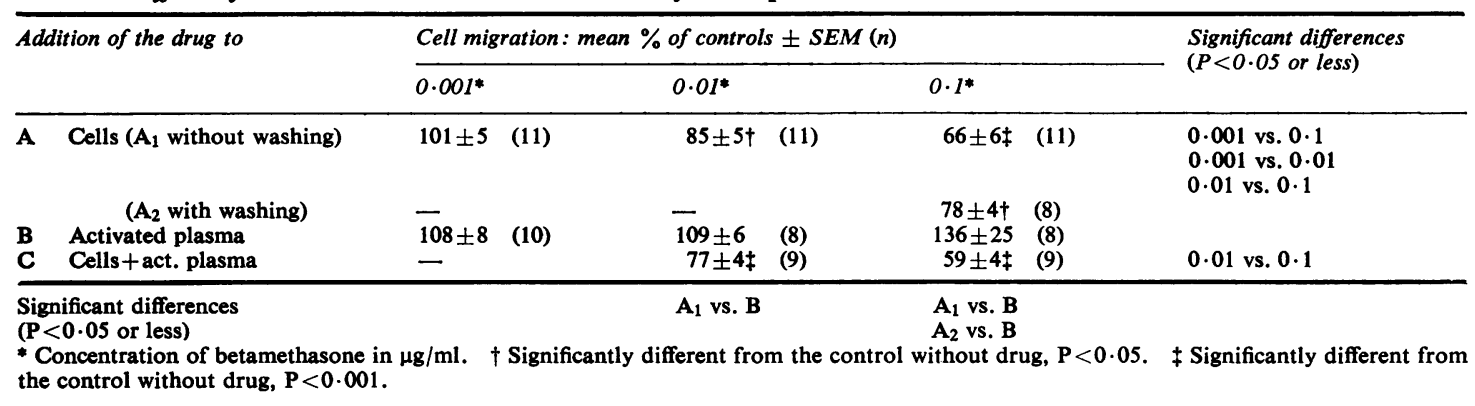

with the same concentrations of betamethasone did not induce any consistent changes in the chemoattractant (experiment $B$ ). When the 2 highest concentrations of the drug were added to both cells and plasma (experiment $\mathrm{C}$ ) a reduction to $77 \%$ and $59 \%$ was observed. These results do not differ significantly from those obtained when incubating the cells alone.

Lysin acetylsalicylate. Table 3 shows that no significant change was observed when incubating either the cells or the plasma with 3 concentrations of this drug. A trend towards a stimulation of chemotaxis was seen after incutating the cells with this substance, but the response was not dose-dependent.

\section{MIGRATION WITHOUT CHEMOATTRACTANT ('RANDOM MIGRATION')}

When no chemoattractant was introduced into the lower compartment, no definite effect was measured, whichever drug was used (Table 4). Preincubation with each of the drugs did not reduce cell migration. A moderate increase in the number of migrating cells was found when betamethasone and acetylsalicylate were added to the lower compartment, but this did not prove to be dose-related.

\section{Discussion}

The most pertinent conclusion that we can draw from these data concerns the effect of a gold salt;

Table 3 Effect of lysin salicylate on the chemotaxis of neutrophils

\begin{tabular}{|c|c|c|c|}
\hline \multirow{2}{*}{$\begin{array}{l}\text { Addition of the } \\
\text { drug to }\end{array}$} & \multicolumn{3}{|c|}{ Cell migration: mean $\%$ of controls $\pm S E M(n)$} \\
\hline & $0.015^{*}$ & $0.15^{*}$ & $1 \cdot 5^{*}$ \\
\hline $\begin{array}{l}\text { A Cells } \\
\text { B Activated plasma }\end{array}$ & $\begin{array}{r}107 \pm 13 \text { (12) } \\
92 \pm 10 \quad(8)\end{array}$ & $\begin{array}{l}125 \pm 22(12) \\
107 \pm 9\end{array}$ & $\begin{array}{c}125 \pm 14 \\
94 \pm 4\end{array}$ \\
\hline
\end{tabular}

Table 4 Effect of sodium aurothiopropanol sulphonate, betamethasone and lysine acetylsalicylate on the migration of neutrophils in the absence of chemoattractant ('random migration')

\begin{tabular}{|c|c|c|c|c|}
\hline \multirow{2}{*}{\multicolumn{2}{|c|}{$\begin{array}{l}\text { Addition of the } \\
\text { drug to }\end{array}$}} & \multicolumn{3}{|c|}{ Cell migration : mean $\%$ of controls $\pm S E M(n)$} \\
\hline & & $\begin{array}{l}\text { Na aurothio. } \\
\text { sulf. gold: } \\
3 \mathrm{\mu g} / \mathrm{ml}\end{array}$ & $\begin{array}{l}\text { Betamethasone } \\
0 \cdot 1 \mu / m l\end{array}$ & $\begin{array}{l}A S A \\
0.15 \mathrm{mg} / \mathrm{ml}\end{array}$ \\
\hline $\mathbf{A}$ & $\begin{array}{l}\text { Cells (upper } \\
\text { compart.) } \\
\text { Buffer (lower } \\
\text { compart.) }\end{array}$ & $\begin{array}{c}89 \pm 6 \\
120 \pm 22\end{array}$ & $\begin{array}{c}95 \pm 10(11) \\
165 \pm 16^{*}(8)\end{array}$ & $\begin{array}{c}96 \pm 8 \quad(10) \\
144 \pm 16^{*}(8)\end{array}$ \\
\hline
\end{tabular}

* Significantly different from the control without drug: $\mathbf{P}<0.005$.

when both neutrophils and plasma were preincubated with this compound, that is, in a situation which more or less mimics the in vivo conditions, an impressive reduction of chemotactic function was observed. Many mechanisms have been cited as explanations of the action of gold salts. They have been shown to inhibit lysosomal enzymes in guinea-pigs macrophages (Persellin and Ziff, 1966), to inhibit the migration and phagocytosis of rat macrophages in experimental inflammation (Jessop et al., 1973; Vernon-Roberts et al., 1973), to interfere with prostaglandin metabolism (Stone et al., 1975), to inhibit the human lymphocyte response induced by mitogens (Lipsky and Ziff, 1977) and, more recently, to inhibit the chemotactic responsiveness of human blood monocytes (Ho et al., 1978).

The reduction of chemotactic function which was observed in our experiment has been obtained with gold concentrations covering the range of therapeutic levels measured in patients suffering from rheumatoid arthritis and treated with gold salts. Mowat (1978), when introducing another gold compound into the upper compartment only, reported a definite inhibition of the neutrophils' chemotaxis. In spite of some technical differences the magnitude of the effect he observed was quite similar to that found in the present study when the 
gold salt was added to the cells only, But there is strong evidence that the overall inhibition found in our study is based on 2 distinct effects: one on the cellular and the other on the plasma component of the chemotactic function. This is in agreement with the results reported by Ward et al. (1975) on the inhibition by gold salt of the chemotaxis of rabbit neutrophils obtained from peritoneal exudates.

How this compound interferes with the cell capacity to respond to a chemotactic stimulus by actively migrating toward a chemical gradient remains to be determined. But the reduction of the capacity of plasma to attract neutrophils might be related to at least 2 effects previously reported by other groups. On the one hand Schultz et al. (1974) reported that in human serum therapeutic concentrations of gold inactivated the complement components $\mathrm{C}_{1}$ and $\mathrm{C}_{1}$ s. So gold salts might decrease the chemoattractive capacity of plasma by interfering with the complement pathway, thus lowering the generation of complement components specifically devoted to chemotactic stimuli such as $\mathrm{C}_{5}$ and $C_{3}$. On the other hand aggregated immunoglobulin or immunocomplexes are thought to generate the inflammatory mediators of this complement system in rheumatoid synovitis (Ruddy and Austen, 1973). Aggregated immunoglobulin was also the substance chosen in our experiments for generating chemotactic activity in plasma. Gerber (1971) has reported that gold thiomalate inhibited the heat aggregation of human gammaglobulin. In our experiments the gold salt was added to the plasma at the same time as the aggregated gammaglobulin and not during the gammaglobulin heating phase. However, it cannot be excluded that gold salt decreased the effect of the activator itself, thus decreasing the generation of chemotactic factors.

These results obtained in vitro need to be confirmed by in vivo studies, such as skin window techniques, However, they suggest that reduction in neutrophil chemotaxis might be an important additional mechanism in the action of gold salts.

The results of studies on the effect of corticosteroids on chemotaxis are contradictory, depending on the origin of the cells, the animal species and the type of steroid studied (Ward, 1971; Peters et al., 1972; Sneiderman and Wilson, 1975; Majeski and Alexander, 1976; Shea and Morse, 1978). Using a filter technique Majeski and Alexander (1976) have reported that the chemotactic function of human neutrophils was not influenced by various corticosteroids except for very high concentrations of hydrocortisone $(1000 \mu \mathrm{g} / \mathrm{ml})$, which produces a $30 \%$ inhibition of the function. Shea and Morse (1978) recently reported a $40 \%$ decrease in human neutrophil chemotaxis with $2 \cdot 5 \mu \mathrm{g} / \mathrm{ml}$ hydrocortisone, i.e., a concentration which can be compared to $0 \cdot 1$ $\mu \mathrm{g} / \mathrm{ml}$ betamethasone. However, mobilisation of leucocytes measured by a skin chamber technique in man was found to be decreased by prednisone and increased by dexamethasone (Peters et al., 1972). In our study, betamethasone inhibited the chemotactic functon only by acting on the neutrophil. Furthermore this inhibition was weak (less than $50 \%$ ). But we used low drug concentrations, which correspond to the estimated blood level reached in man after ingestion of $4 \mathrm{mg}$ of betamethasone. So corticosteroids seem less effective than gold salts in reducing the chemotactic function of neutrophils; perhaps they might act by different mechanisms. Gallin et al. (1975) have suggested one possible mechanism: the chemotactic factor $\mathrm{C}_{5}$ a decreased the negative surface charge of human granulocytes; hydrocortisone inhibited both the effect of $\mathrm{C}_{5} \mathrm{a}$ on granulocyte surface charge and its chemotactic activity, suggesting that steroids act on the cell surface.

In our study aspirin was found to have no influence on the chemotaxis of neutrophils in either therapeutic concentrations as observed in aspirin treated rheumatoid patients $(0.15 \mathrm{mg} / \mathrm{ml})$ or at a highly toxic blood level $(1.5 \mathrm{mg} / \mathrm{ml})$. These data are in agreement with those obtained in vitro by Ward (1971) and in vivo by Mowat and Baum (1971) in patients suffering from rheumatoid arthritis treated with aspirin. Technical variations might account for the discrepancies between our data and those of Magliulo et al. (1977), who have reported an inhibition of the chemotactic function with this drug. It is possible that aspirin decreases leucocyte adherence to the filters, which would result in an artificially low estimation of chemotaxis in experiments such as Magliulo et al. in which the migrated leucocytes were not counted on the bottom of the lower compartment. Thus for drug assays it is probably important to count the leucocytes both on the filter and on the bottom of the lower compartment.

Migration without chemoattractant was not clearly influenced by the drug tested. Because of the very small number of cells usually found in the lower compartment after migration a filter technique is probably not the best one for the study of this type of migration. It does not enable us to draw conclusions from the discrepancies observed between the action of anti-inflammatory drugs on the migration with and without chemoattractant. However, the normal or enhanced migration observed without chemoattractant might be considered as an additional sign of intact cell viability after drug preincubation.

This study suggests that drugs effective in rheu- 
matoid arthritis behave differently with regard to their inhibitory action on the chemotactic function of neutrophils. Gold salts, which exhibit the longest therapeutic action in rheumatoid arthritis, are the most effective, corticosteroids appeared to be moderately effective and aspirin not at all. This also seems to indicate that there is probably not 1 sole mechanism of action for anti-inflammatory drugs.

This study was supported in part by the Swiss Conferation, under proposition of the Commission Fédérale des maladies rhumatismales.

We wish to thank Jeanne-Chantal de Flaugergues, Margit Schubert, and Michèle Isely-Ochsner for skilful technical assistance, Professor Jean-Louis Schelling for editorial assistance, and Dr Bernard Wuilleret, chief of the Centre de Transfusion Sanguine, Lausanne, for providing us with blood from normal donors.

\section{References}

Baum, J. (1975). Chemotaxis in Human Disease. The Phagocytic Cell in Host Resistance. Edited by J. A. Bellanti and D. H. Dayton. Raven Press: New York.

Boyden, S. (1962). The chemotactic effect of mixtures of antibody and antigen on polymorphonuclear leucocytes. Journal of Experimental Medicine, 115, 453-466.

Frei, P. C., Baisero, M. H., and Ochsner, M. (1974). Chemotaxis of human polymorphonuclears in vitro: II Technical study. Journal of Immunological Methods, 5, 375-386.

Gallin, J. I., Durocher, J. R., and Kaplan, A. P. (1975). Interaction of leukocyte chemotactic factors with the cell surface. Journal of Clinical Investigation, 55, 967-974.

Gerber, D. A., (1971). Inhibition of heat-aggregation of human gammaglobulin by histidine, gold thiomalate and penicillamine. Arthritis and Rheumatism, 14, 383.

Ho, P. P. K., Young, A. L., and Southard, G. L. (1978). Methyl ester of $\mathrm{N}$-formylmethionyl-leucyl-phenylalanine. Chemotactic responses of human blood monocytes and inhibition of gold compounds. Arthritis and Rheumatism, 21, 133-136.

Jessop, J. D., Vernon-Roberts, B., and Harris, J. (1973). Effects of gold salts and prednisolone on inflammatory cells. I. Phagocytic activity of macrophages and polymorphs in inflammatory exudates studied by a 'skin window' technique in rheumatoid and control patients. Annals of the Rheumatic Diseases, 32, 294-300.

Keller, H. U., Wilkinson, P. C., Abercrombie, M., Becker, E. L., Hirsch, J. G., Miller, M. E., Ramsey, W. S., and Zigmond, S. H. (1977). A proposal for the definition of terms related to locomotion of leucocytes and other cells. Clinical and Experimental Immunology, 27, 377-380.
Lipsky, P. E., and Ziff, M. (1977). Inhibition of antigen- and mitogen-induced human lymphocyte proliferation by gold compounds. Journal of Clinical Investigation, 59, 455-466.

Magliulo, E., Benzi-Cipelli, R., Lago, A., Azzini, M., Magni, E., Gallico, S., and Sacchetti, G. (1977). Inhibiting effect of non-steroidal anti-inflammatory drugs on the chemotaxis of human leukocytes in vitro. International Journal of Clinical Pharmacology, 15, 417-418.

Majeski, J., and Alexander, J. W. (1976). The steroid effect on the in vitro human neutrophil chemotactic response. Journal of Surgical Research, 21, 265-268.

Mowat, A. G. (1978). Neutrophil chemotaxis in rheumatoid arthritis. Effect of D-penicillamine, gold salts, and levamisole. Annals of the Rheumatic Diseases, 37, 1-8.

Mowat, A. G. and Baum, J. (1971). Chemotaxis of polymorphonuclear leukocytes from patients with rheumatoid arthritis. Journal of Clinical Investigation, 50, 2541-2549.

Persellin, R. H., and Ziff, M. (1966). The effect of gold salt on lysosomal enzymes of the peritoneal macrophage. Arthritis and Rheumatism, 9, 57-65.

Peters, W. P., Holland, J. F., Senn, H. J., Rhomberg, W. and Banerjee, T. (1972). Corticosteroid administration and localized leukocyte mobilization in man. New England Journal of Medicine, 282, 342-345.

Ruddy, S., and Austen, K. F. (1973). Activation of the complement system in rheumatoid synovitis. Federation Proceedings, 32, 134-137.

Schultz, D. R., Volanakis, J. E., Arnold, P. I., Gottlieb, N. L., Sakai, K., and Stroud, R. M. (1974). Inactivation of $C_{1}$ in rheumatoid synovial fluid, purified $C_{1}$ and $C_{1}$ esterase by gold compounds. Clinical and Experimental Immuno$\log y, 17,395-406$.

Shea, C., and Morse, E. D. (1978). Inhibition of humate neutrophil chemotaxis by corticosteroids. Annals of Clinical and Laboratory Sciences, 8, 30-33.

Sneiderman, C. A., and Wilson, J. W. (1975). Effects of corticosteroids on complement and the neutrophilic polymorphonuclear leukocyte. Transplantation Proceedings, 7, 41-48.

Stone, K. J., Mather, S. J., and Gibson, P. P. (1975). Selective inhibition of prostaglandin biosynthesis by gold salts and phenylbutazone. Prostaglandins, 10, 241-251.

Vernon-Roberts, B., Jessop, J. D., and Doré, J. (1973). Effects of gold salts and prednisolone on inflammatory cells. II. Suppression of inflammation and phagocytosis in the rat. Annals of the Rheumatic Diseases, 32, 301-307.

Ward, P. A. (1966). The chemosuppression of chemotaxis. Journal of Experimental Medicine, 124, 209-225.

Ward, P. A. (1971). Leukotactic factors in health and disease. American Journal of Pathology, 64, 521-530.

Ward, P. A., Goldschmidt, P., and Greene, N. D. (1975) Suppressive effects of metal salts on leukocyte and fibroblastic function. Journal of the Reticuloendothelial Society, 18, 313-321. 